\title{
论文
}

\section{不同价态无机磷在金属氧化物表面吸附的 第一性原理研究}

衡忠暄 ${ }^{1}$, 单超 ${ }^{1,2^{*}}$, 花铭 ${ }^{1,2}$, 潘丙才 ${ }^{1,2}$

1. 南京大学环境学院, 污染控制与资源化研究国家重点实验室, 南京 210023;

2. 南京大学环境纳米技术研究中心, 南京 210023

*E-mail: shanchao@nju.edu.cn

收稿日期: 2020-07-08; 接受日期: 2020-09-07; 网络版发表日期: 2021-03-12

国家重点研发计划(编号: 2017YFE0107200)和中央高校基本科研业务费专项(编号: 14380145)资助项目

摘要磷是控制水体富营养化的关键限制因子. 目前主流的除磷技术处理正磷酸盐相对成熟, 但次/亚磷酸盐的 深度去除尚未引起充分关注. 金属氧化物是一类常用的去除正磷酸盐的吸附剂, 探究不同价态无机磷在金属氧化 物表面吸附行为与机理具有重要研究意义. 本文构建了晶型和无定形 $\mathrm{Fe}_{2} \mathrm{O}_{3}, \mathrm{ZrO}_{2}, \mathrm{La}_{2} \mathrm{O}_{3}$ 体相与基底模型, 基于第 一性原理, 计算了次磷酸盐、亚磷酸盐、正磷酸盐在上述基底上的吸附构型及吸附能. 结果表明, 金属氧化物与 次磷酸盐、亚磷酸盐、正磷酸盐主要生成 $\mathrm{M}-\mathrm{O}-\mathrm{P}$ 键 $(\mathrm{M}$ 为金属原子)和氢键 $(\mathrm{O}-\mathrm{H})$. 根据吸附能比较, 正磷酸盐在 金属氧化物表面主要为化学吸附, 且吸附能普遍大于次/亚磷酸盐在相应基底的吸附能(0.7 6.3倍), 表明正磷酸盐 易被金属氧化物专属吸附, 而次/亚磷酸盐吸附能较小，相对易受到共存离子竞争干扰。无定形金属氧化物对 次/亚/正磷酸盐的吸附能力优于晶型金属氧化物(吸附能均值为 3.1 倍), 三种金属氧化物吸附能力由弱到强的顺序 为 $\mathrm{Fe}_{2} \mathrm{O}_{3}<\mathrm{ZrO}_{2}<\mathrm{La}_{2} \mathrm{O}_{3}$, 次/亚/正磷酸盐在氧化镧基底上的吸附能为氧化铁/锆的 2.0 47.5 倍, 表明了 $\mathrm{La}$ 与 $\mathrm{PO}_{x}$ 基团之 间强烈的成键作用. 本文可为深度除磷技术选择和开发提供参考.

关键词吸附, 磷形态, 金属氧化物, 密度泛函理论, 吸附能

\section{1 引言}

水体富营养化是全球关注的环境问题 ${ }^{[1]}$. 磷是水 体富营养化的限制因子，对磷的污染控制是防止富营 养化的关键. 在氮充足的条件下, 磷酸盐浓度仅为 $0.03 \sim 0.04 \mathrm{mg} / \mathrm{L}$ 时, 澡类比增长速率即迅速增加 ${ }^{[2]}$. 为 遏制水体富营养化, 发展深度除磷技术并探究相关机
理具有重要意义.

水中磷大体可分为无机磷和有机磷, 在市政污水 和工业废水生化尾水中通常以无机磷为主. 水中无机 磷主要包括次磷酸盐、亚磷酸盐、正磷酸盐( 以下简 称次磷、亚磷、正磷). 次磷的 $\mathrm{pKa}$ 为 1.2 , 亚磷的 $\mathrm{pKa}_{1}$, $\mathrm{pKa}_{2}$ 分别为 1.3 和 6.6 , 正磷的 $\mathrm{pKa}_{1}, \mathrm{pKa}_{2}, \mathrm{pKa}_{3}$ 分别为 $2.1,7.2,12.4$. 在近中性 $\mathrm{pH}$ 的条件下, 次磷、亚磷、正

引用格式: 衡忠暄, 单超, 花铭, 等. 不同价态无机磷在金属氧化物表面吸附的第一性原理研究. 中国科学: 技术科学, 2021, 51: 591-600 Heng Z X, Shan C, Hua M, et al. Adsorption of inorganic phosphorus of different valences on metal oxides: A first-principles study (in Chinese). Sci Sin Tech, 2021, 51: 591-600, doi: 10.1360/SST-2020-0281 
磷主要形态分别为 $\mathrm{H}_{2} \mathrm{PO}_{2}{ }^{-}, \mathrm{H}_{2} \mathrm{PO}_{3}{ }^{-}$和 $\mathrm{HPO}_{3}{ }^{2-}, \mathrm{HPO}_{4}{ }^{2-}$ 和 $\mathrm{H}_{2} \mathrm{PO}_{4}^{-}$.

水中无机磷以正磷为主, 徐伟勇 ${ }^{[3]}$ 在城市污水处 理厂生化尾水中检测出溶解性正磷占总磷的 $76.8 \%$. 次磷、亚磷比例较小, 在自然水体中浓度通常较低 ${ }^{[4,5]}$, 但次/亚磷在化学镀等工业废水中浓度较高且产生量 $大^{[6]}$, 浓度可达 $2.5 \mathrm{~g} / \mathrm{L}^{[7]}$.

随着水环境和污废水排放标准的提高, 次/亚磷将 成为深度除磷的瓶颈之一, 但目前次/亚磷的深度去除 尚未引起充分的重视. 因此, 需要发展针对次/亚磷的 高效深度处理技术.

磷的处理方法主要包括生物法和化学沉淀法, 出 水总磷可低于 $0.5 \mathrm{mg} / \mathrm{L}^{[8 \sim 10]}$. 深度除磷方法包括吸附法 等, 出水总磷可达到约 $0.2 \sim 0.3 \mathrm{mg} / \mathrm{L}^{[11,12]}$. 然而, 这些处 理技术通常是用于正磷的处理, 对于次/亚磷的处理效 果比较有限. 对生物除磷技术而言, 很多微生物难以利 用次/亚磷作为磷源 ${ }^{[13,14]}$, 反应体系稳定程度不高 ${ }^{[15]}$. 化 学沉淀法对次/亚磷效果往往不佳, 例如, $\mathrm{Ca}\left(\mathrm{H}_{2} \mathrm{PO}_{2}\right)_{2}$ 的 溶解度高达 $16.7 \mathrm{~g} / 100 \mathrm{~g}$ 水. 吸附除磷方面, 正磷酸盐在 氧化铁 ${ }^{[16]}$ 、氧化锆 ${ }^{[17]}$ 、氧化镧 ${ }^{[18]}$ 等诸多金属氧化物 表面普遍表现为专属吸附, 因而在共存离子等复杂基 质背景下仍可表现出良好的磷吸附性能; 但目前常见 金属氧化物吸附次/亚磷的强度和机理尚不清楚, 有必 要进行深入探索. 由于正磷的去除相对成熟, 将次/亚 磷氧化为正磷是一种有效的预处理策略; 然而水中共 存离子等基质十分复杂, 大量共存基质会竞争氧化剂 或氧化活性物种, 为提高氧化剂的有效利用率, 这一 过程通常需要借助具有选择性氧化功能的催化剂. 吸 附是绝大多数非均相催化反应的先决步骤 ${ }^{[19]}$, 因此, 研究不同价态无机磷在金属氧化物表面的吸附机理除 了对吸附除磷技术开发具有基础指导意义以外，还可 为高效催化剂的搜寻和设计提供理论依据 ${ }^{[19,20]}$.

吸附作用是吸附质分子和吸附剂基底之间的相互 作用，其作用强弱本质上是由双方搭建成吸附构型的 原子的电子结构所决定的. 吸附能是定量描述吸附结 合强度的关键指标, 对复杂共存基质条件下目标污染 物吸附效能的预测具有重要意义, 可为吸附剂筛选、 搜寻和设计提供科学指导. 然而, 通过实验研究获得 微观吸附能信息的方法往往依赖经验公式, 受到各方 面实验条件干扰较大, 不确定度较高, 难以准确反映 相关吸附机理. 近年来, 计算化学迅速发展, 运用密度
泛函理论研究物质的界面吸附行为和机理已成为高效 吸附剂和催化剂搜寻和设计的重要科学方法. 本文选 取对正磷具有良好吸附能的氧化铁、氧化锆和氧化镧 作为典型金属氧化物代表, 分别考虑晶型和无定形两 种形式, 通过模型构建、结构优化和高精度单点能计 算, 得到次磷、亚磷、正磷在上述界面的吸附构型和 吸附能信息，从而探讨不同价态无机磷在金属氧化物 表面的吸附特性与机理. 本文可为深度除磷水处理技 术开发和选择提供科学指导, 也可为不同价态无机磷 的环境界面行为和环境归趋等方面的研究提供参考.

\section{2 研究方法}

\section{1 基底与吸附质模型构建}

选取的晶型金属氧化物为 $\alpha-\mathrm{Fe}_{2} \mathrm{O}_{3}, \mathrm{~m}-\mathrm{ZrO}_{2}$, $\mathrm{h}-\mathrm{La}_{2} \mathrm{O}_{3}$, 构型导入自Materials Studio内置数据库. 三 种晶型金属氧化物基底模型主要经过扩胞、切割晶 面、构建真空层三个步骤构建. 切割晶面方向选取三 种晶型金属氧化物的主要衍射晶面方向, 其中 $\mathrm{Fe}_{2} \mathrm{O}_{3}$ 切 割(104)和(110)晶面, $\mathrm{ZrO}_{2}$ 切割(111)晶面, $\mathrm{La}_{2} \mathrm{O}_{3}$ 切割 (101)晶面; 所有真空层厚度均设置为 $15 \AA$.

无定形金属氧化物基底模型的构建分为两个阶 段：第一阶段采用从头算分子动力学模拟构建无定形 金属氧化物体相模型，第二阶段构建基底表面模型. 第一阶段的构建策略为熔化-淬冷 ${ }^{[21]}$, 从头算分子动力 学过程在Vienna Ab-initio Simulation Package (VASP) 中进行模拟, $\mathrm{Fe}_{2} \mathrm{O}_{3}, \mathrm{ZrO}_{2}, \mathrm{La}_{2} \mathrm{O}_{3}$ 的“熔化”温度分别设 置为 $3000,5000,4000 \mathrm{~K}$, 采用正则系综, 动力学模拟时 间为 $20 \mathrm{ps}$; 淬冷温度均为 $0 \mathrm{~K}$, 动力学模拟时间为 $20 \mathrm{ps,}$ 并在淬冷过程后进行结构优化. 第一阶段的构建结果 使用VESTA ${ }^{[22]}$ 中的X射线衍射(XRD)模拟和Materials Studio的径向分布函数(RDF)计算进行验证. 第二阶段 为切割表面、构建真空层, 参照晶型氧化物的方法, 其 中在切割方向上, 虽然无定形金属氧化物无晶面概念, 但为与相应晶型金属氧化物模型保持一致，仍沿相同 的方向切割.

水中次/亚/正磷在通常 $\mathrm{pH}$ 条件下以阴离子形式存 在. 在第一性原理计算中并无对应电荷的客观数学描 述, 无法为具体原子或原子团指定所带电荷数量; 如 设定整体背景电荷依靠电子自洽过程进行局域化, 所 得电荷局域结果在多种变价元素共存条件下可能与实 
际情况存在偏差．在不影响体系主要化学作用的前提 下，第一性原理计算中经常采用氢原子饱和、添加噟 氢针化极性表面等方法. 本文主要注重搜寻具有专属 吸附作用的界面，而考虑到质子化程度对化学吸附作 用的影响较小，本文构建的次磷、亚磷、正磷三种吸 附质为电中性的 $\mathrm{H}_{3} \mathrm{PO}_{2}, \mathrm{H}_{3} \mathrm{PO}_{3}, \mathrm{H}_{3} \mathrm{PO}_{4}$ 分子, 将其置于 边长为 $10 \AA$ 的立方单元中.

对上述构建的基底与吸附质模型均进行结构优化 与高精度单点能计算, 结构优化为后续构建吸附模型 提供基础，高精度单点能是后续差减法计算吸附能的 依据.

\section{2 吸附能计算}

吸附初始构型为基底与吸附质优化后构型的组 合, 按照界面配体交换经典理论 ${ }^{[23]}$ 构建. 对吸附构型 依次进行结构优化和计算高精度单点能.

吸附能计算如式(1)所示:

$E_{\mathrm{ad}}=E_{\mathrm{MO}+\mathrm{P}}-E_{\mathrm{MO}}-E_{\mathrm{P}}$,

其中, $E_{\mathrm{ad}}$ 表示吸附能, $E_{\mathrm{MO}+\mathrm{P}}$ 表示吸附优化后构型的总 能量, $E_{\mathrm{MO}}$ 表示金属氧化物基底的能量, $E_{\mathrm{P}}$ 表示吸附质 分子(次/亚/正磷)的能量.

\section{3 计算参数}

使用VASP软件进行结构优化 ${ }^{[24]}$, 采用投影缀加平 面波噟势以及广义梯度近似中的Perdew-Burke-Ernzerhof交换相关泛函 ${ }^{[25]}$. 部分占据状态选用高斯方法, 展宽设为 $0.05 \mathrm{eV}$, 离子弛豫选择共轭梯度法, 截断能 设为 $400 \mathrm{eV}$, 电子自洽能量收玫标准设为 $10^{-5} \mathrm{eV}$, 离子 弛豫收玫标准设为原子力小于 $0.05 \mathrm{eV} \AA^{-1} . k$ 点采样选 择以 $\Gamma$ 点为中心的 $3 \times 3 \times 1$ 的 $k$ 点网格. 高精度单点能计 算的电子自洽能量收玫标准为 $10^{-6} \mathrm{eV}$, 其他基本参数 同结构优化.

\section{3 结果与讨论}

\section{1 模型构建}

\subsection{1晶型金属氧化物基底模型构建}

$\alpha-\mathrm{Fe}_{2} \mathrm{O}_{3}, \mathrm{~m}-\mathrm{ZrO}_{2}, \mathrm{~h}-\mathrm{La}_{2} \mathrm{O}_{3}$ 三种晶型金属氧化物基 底优化后构型如图1所示. 优化后的 $\mathrm{Fe}-\mathrm{O}$ 键和 $\mathrm{La}-\mathrm{O}$ 键 长比优化前总体略有缩短. 优化前, $\mathrm{Fe}-\mathrm{O}$ 键长为
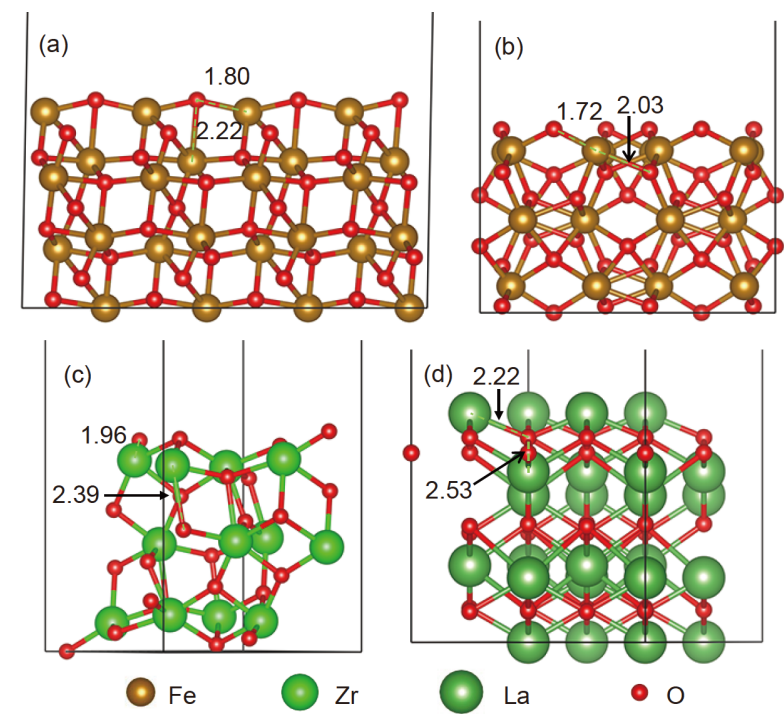

图 1 (网络版彩图)晶型金属氧化物基底优化后构型. (a) $\alpha-\mathrm{Fe}_{2} \mathrm{O}_{3}$ (104)晶面; (b) $\alpha-\mathrm{Fe}_{2} \mathrm{O}_{3}$ (110)晶面; (c) $\mathrm{m}-\mathrm{ZrO}_{2}$ (111) 晶面; (d) h- $\mathrm{La}_{2} \mathrm{O}_{3}$ (101) 晶面; 键长单位: $\AA$

Figure 1 (Color online) Optimized configurations of crystal metal oxide substrates. (a) $\alpha-\mathrm{Fe}_{2} \mathrm{O}_{3}$ (104) facet; (b) $\alpha-\mathrm{Fe}_{2} \mathrm{O}_{3}$ (110) facet; (c) $\mathrm{m}-\mathrm{ZrO}_{2}$ (111) facet; (d) $\mathrm{h}-\mathrm{La}_{2} \mathrm{O}_{3}$ (101) facet; bond length in $\AA$.

$1.96 \AA$ 或 $2.09 \AA ; \mathrm{La}-\mathrm{O}$ 键长有 $2.37,2.46,2.73 \AA$ 三种. 优化后, (104)晶面和(110)晶面的 $\mathrm{Fe}-\mathrm{O}$ 键长分别在 $1.80 \sim 2.22$ 和 $1.7 \sim 2.03 \AA$ 范围; $\mathrm{La}-\mathrm{O}$ 键长范围为 $2.22 \sim$ $2.53 \AA$, 大部分在2.22 2.39 $\AA$ 之间.

\section{1 .2 无定形金属氧化物基底模型构建}

无定形金属氧化物体相模型依次经过熔化、淬冷 和结构优化的具体构建过程如图2所示. 相较对应的晶 型金属氧化物，无定形金属氧化物体相模型中金属原 子的配位数减小, $\alpha-\mathrm{Fe}_{2} \mathrm{O}_{3}, \mathrm{~h}-\mathrm{La}_{2} \mathrm{O}_{3}, \mathrm{~m}-\mathrm{ZrO}_{2}$ 中 Fe, $\mathrm{La}$, $\mathrm{Zr}$ 的配位数分别为 $6,7,7$, 相应的无定形金属氧化物中 部分 $\mathrm{Fe}, \mathrm{La}, \mathrm{Zr}$ 的配位数分别为 $5,6,6$, 即出现缺陷配位 现象.

对上述构建的无定形金属氧化物体相模型，本文 采用XRD模拟和RDF计算与相应晶型金属氧化物进 行了对比验证, 结果分别如图3所示.

XRD计算结果(图3(a))中, 三种晶型金属氧化物的 主要衍射峰为: $\alpha-\mathrm{Fe}_{2} \mathrm{O}_{3}$ 特征峰出现在 $2 \theta=33.21^{\circ}$, $35.63^{\circ}$ 处, $\mathrm{m}-\mathrm{ZrO}_{2}$ 的特征峰出现在 $2 \theta=29.95^{\circ}$ 处, $\mathrm{h}-\mathrm{La}_{2} \mathrm{O}_{3}$ 在 $2 \theta=28.20^{\circ}, 31.49^{\circ}$ 处呈现特征峰; 三种晶型 金属氧化物RDF图谱(图3(b)) 呈现的规律的尖峰排布 表明相应晶型的长程有序结构. 与晶型氧化物的XRD 

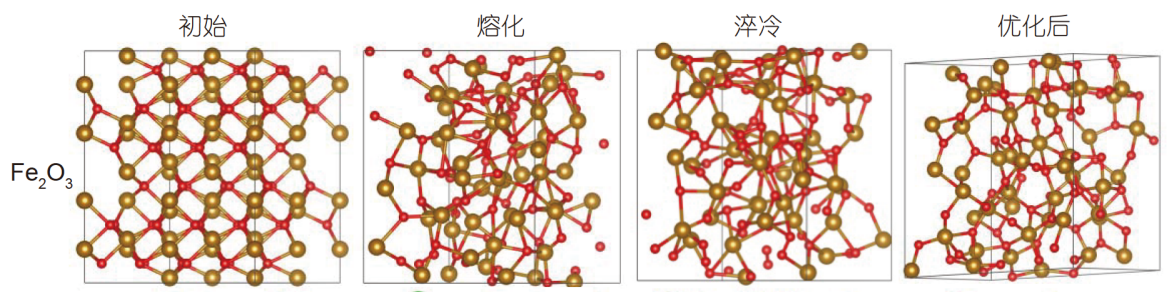

$\mathrm{ZrO}_{2}$
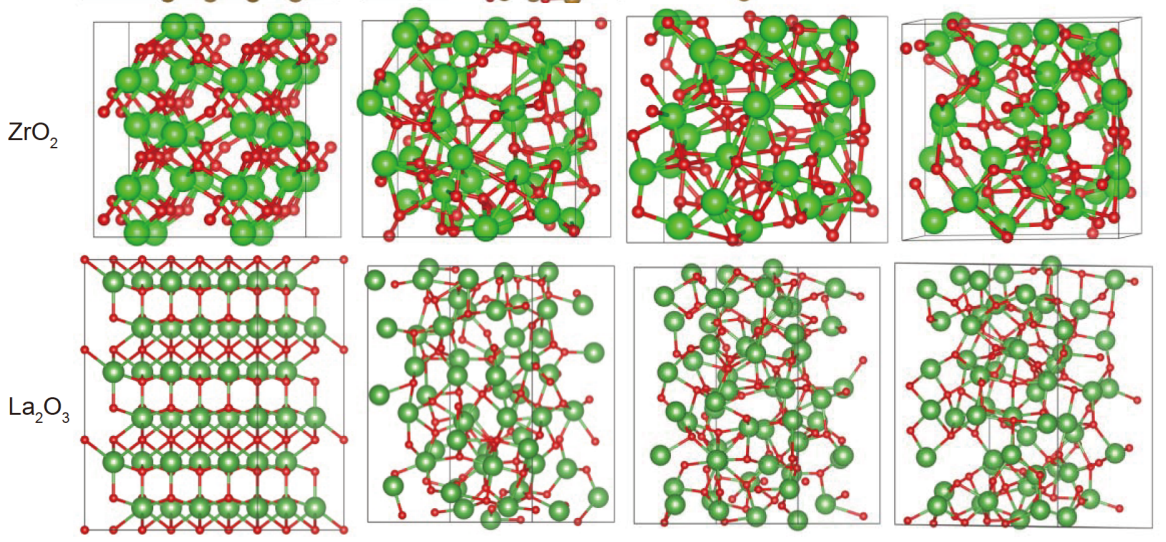

$\odot \mathrm{Fe}$

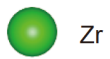

La

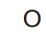

图 2 (网络版彩图)无定形金属氧化物体相模型构建过程

Figure 2 (Color online) Construction process of bulk models of amorphous metal oxide substrates.
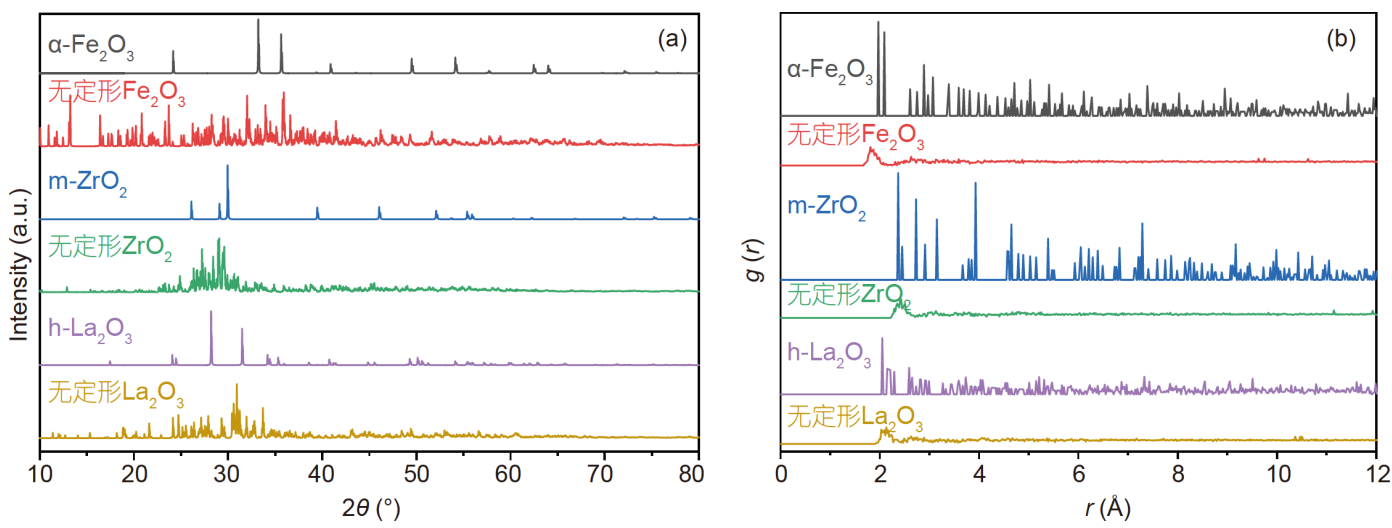

图 3 (网络版彩图)晶型与无定形金属氧化物体相模型的模拟XRD谱图(a)和径向分布函数(RDF)图(b)

Figure 3 (Color online) XRD spectra (a) and radial distribution function (RDF) (b) of the bulk models of crystal and amorphous metal oxides.

和RDF图谱明显不同，构建的三种无定形金属氧化物 的模拟XRD图谱虽并未完全无定形化，但上述晶型氧 化物的主要特征衍射峰已消失或偏移，未能完全无定 形化的主要原因是构建的周期性结构单元(对应晶型 的“超晶胞”概念)受限于计算能力不能无限扩大，仍存 在较微弱的周期性; RDF图中无定形金属氧化物第一 配位壳层峰宽化，径向距离超过 3.0 后峰逐渐消失， 表示长程有序结构被破坏，在吸附行为第一性原理研
究的尺度上已可以代表无定形氧化物的特性，表明三 种金属氧化物无定形体相模型构建成功.

经过切割表面、加真空层和结构优化后构建所得 的三种无定形金属氧化物基底的表面模型如图4所示. 与无定形金属氧化物体相模型相同，原子排布表现为 无序结构，无定形 $\mathrm{Fe}_{2} \mathrm{O}_{3}$ 的 $\mathrm{Fe}-\mathrm{O}$ 键比 $\alpha-\mathrm{Fe}_{2} \mathrm{O}_{3}$ 的 $\mathrm{Fe}-\mathrm{O}$ 键 明显缩短 $(1.7 \sim 1.9 \AA)$, 无定形 $\mathrm{ZrO}_{2}$ 与 $\mathrm{La}_{2} \mathrm{O}_{3}$ 的 $\mathrm{M}-\mathrm{O}$ 键长 与相应晶型金属氧化物无明显差异. 

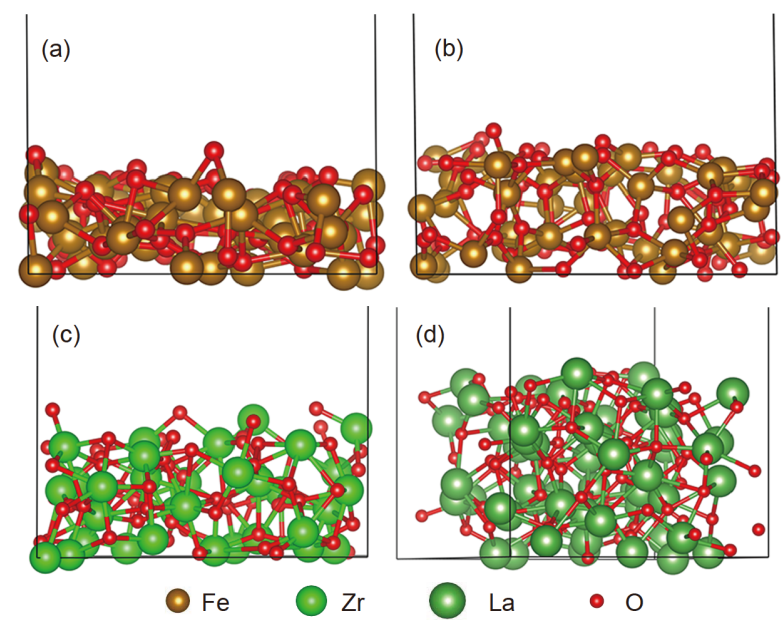

图 4 (网络版彩图)无定形金属氧化物基底优化后构型. (a) 无定形 $\mathrm{Fe}_{2} \mathrm{O}_{3}$ (104)切割方向; (b) 无定形 $\mathrm{Fe}_{2} \mathrm{O}_{3}$ (110)切割方 向; (c) 无定形 $\mathrm{ZrO}_{2}$; (d) 无定形 $\mathrm{La}_{2} \mathrm{O}_{3}$; 键长单位: $\AA$

Figure 4 (Color online) Optimized configurations of amorphous metal oxide substrates. (a) Amorphous $\mathrm{Fe}_{2} \mathrm{O}_{3}$ (104) cleavage direction; (b) amorphous $\mathrm{Fe}_{2} \mathrm{O}_{3}$ (110) cleavage direction; (c) amorphous $\mathrm{ZrO}_{2}$; (d) amorphous $\mathrm{La}_{2} \mathrm{O}_{3}$; bond length in $\AA$.

\subsection{3 不同价态无机磷模型构建}

构建 $\mathrm{H}_{3} \mathrm{PO}_{2}, \mathrm{H}_{3} \mathrm{PO}_{3}, \mathrm{H}_{3} \mathrm{PO}_{4}$ 模型并结构优化, 得到 的构型如图5所示.

\section{2 吸附构型与吸附行为}

$\mathrm{Fe}_{2} \mathrm{O}_{3}, \mathrm{ZrO}_{2}$ 与 $\mathrm{La}_{2} \mathrm{O}_{3}$ 分别吸附不同价态无机磷的 构型经VASP优化后如图6 8所示. 优化后的构型中主 要有两种成键形式：第一种是M-O-P键；第二种主要 表现为氢键. 参与成键的 $\mathrm{H}$ 原子主要是羟基中的 $\mathrm{H}$ 原 子, $\mathrm{P}-\mathrm{H}$ 键中的 $\mathrm{H}$ 原子普遍未参与成键, 除 $\mathrm{h}-\mathrm{La}_{2} \mathrm{O}_{3}$ 吸附 次磷外, $\mathrm{P}-\mathrm{H}$ 键键长无明显变化(小于 $0.03 \AA$ ).

$\mathrm{Fe}_{2} \mathrm{O}_{3}$ 与 $\mathrm{La}_{2} \mathrm{O}_{3}$ 的成键形式比较相似，主要形成1 2 个 $\mathrm{M}-\mathrm{O}$ 键和 $1 \sim 2$ 个氢键. $\mathrm{La}_{2} \mathrm{O}_{3}$ 主要形成 1 个 $\mathrm{La}-\mathrm{O}$ 键并

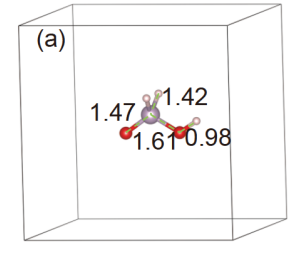

(2) $P$

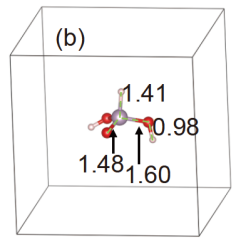

$\odot \mathrm{O} \quad \mathrm{H}$ (c)

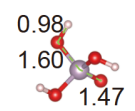

图 5 (网络版彩图)吸附质分子优化后构型. (a) $\mathrm{H}_{3} \mathrm{PO}_{2}$; $\mathrm{H}_{3} \mathrm{PO}_{3}$; (c) $\mathrm{H}_{3} \mathrm{PO}_{4}$; 键长单位: $\AA$

Figure 5 (Color online) Optimized configurations of adsorbate molecules. (a) $\mathrm{H}_{3} \mathrm{PO}_{2}$; (b) $\mathrm{H}_{3} \mathrm{PO}_{3}$; (c) $\mathrm{H}_{3} \mathrm{PO}_{4}$; bond length in $\AA$.

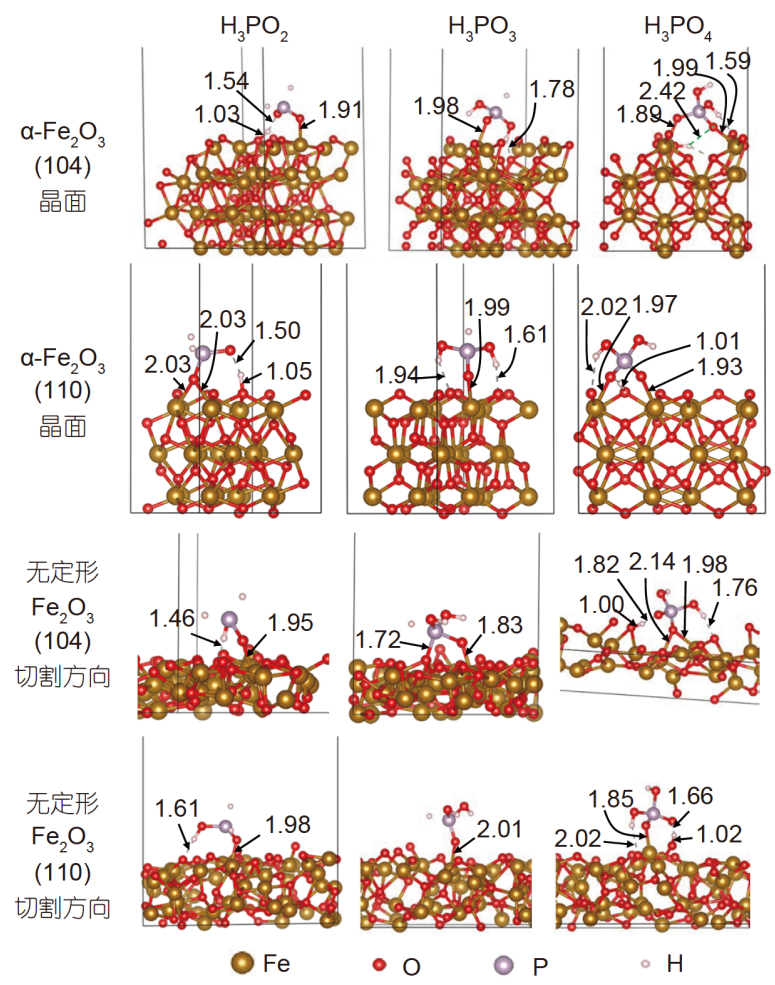

图 6 (网络版彩图) $\mathrm{Fe}_{2} \mathrm{O}_{3}$ 吸附不同价态无机磷优化后构型. 虚线表示氢键; 键长单位: $\AA$

Figure 6 (Color online) Optimized adsorption configurations of inorganic $\mathrm{P}(\mathrm{I} / \mathrm{III} / \mathrm{V})$ on $\mathrm{Fe}_{2} \mathrm{O}_{3}$. Dash lines represent hydrogen bonds; bond length in $\AA$.

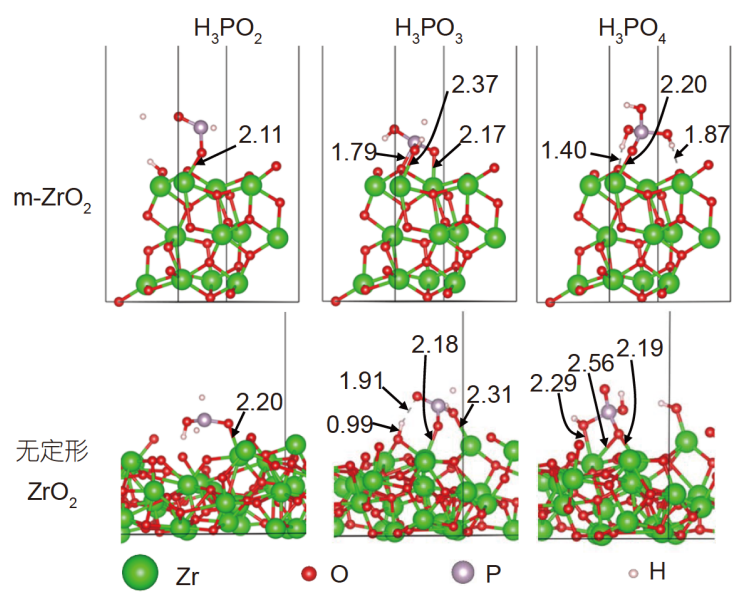

图 7 (网络版彩图) $\mathrm{ZrO}_{2}$ 吸附不同价态无机磷优化后构型. 虚线表示氢键; 键长单位: $\AA$

Figure 7 (Color online) Optimized adsorption configurations of inorganic $\mathrm{P}(\mathrm{I} / \mathrm{III} / \mathrm{V})$ on $\mathrm{ZrO}_{2}$. Dash lines represent hydrogen bonds; bond length in $\AA$.

夺1个 $\mathrm{H}$ 原子. 总体上 $\mathrm{La}_{2} \mathrm{O}_{3}$ 表现出更强的吸附, 其中 $\mathrm{h}-\mathrm{La}_{2} \mathrm{O}_{3}$ 夺去了次磷中 $\mathrm{P}-\mathrm{H}$ 键的 $\mathrm{H}$ 原子, 导致 $\mathrm{P}$ 原子只与 

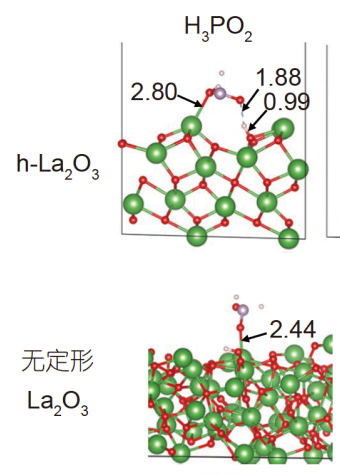

( La
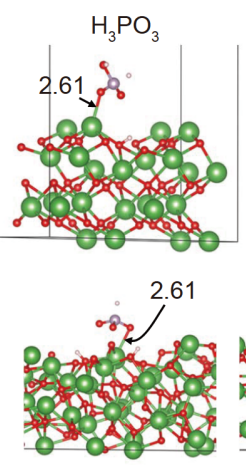

○
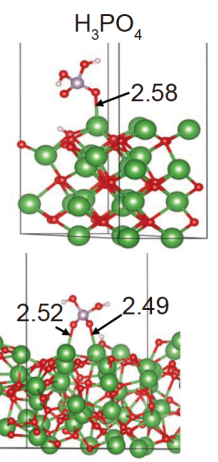

- $\mathrm{H}$
图 8 (网络版彩图) $\mathrm{La}_{2} \mathrm{O}_{3}$ 吸附不同价态无机磷优化后构型 虚线表示氢键; 键长单位: $\AA$

Figure 8 (Color online) Optimized adsorption configurations of inorganic $\mathrm{P}(\mathrm{I} / \mathrm{III} / \mathrm{V})$ on $\mathrm{La}_{2} \mathrm{O}_{3}$. Dash lines represent hydrogen bonds; bond length in $\AA$.

3 个原子成键，结构与其他吸附构型有较大区别， $\mathrm{P}-\mathrm{H}$ 键长度为 $1.47 \AA$, 无 $\mathrm{H}$ 与 $\mathrm{O}$ 相连的 $\mathrm{P}-\mathrm{O}$ 键为 $1.54 \AA$, 有 $\mathrm{H}$ 与 $\mathrm{O}$ 相连的 $\mathrm{P}-\mathrm{O}$ 键为 $1.75 \AA$.

\section{3 吸附能与吸附机理}

根据式(1)计算各吸附情况的吸附能，如表1所示. 除无定形 $\mathrm{Fe}_{2} \mathrm{O}_{3}$ (104)切割方向吸附亚磷外，各个吸附 能均为负值, 说明所考察的吸附均为热力学自发过程. 将各吸附能计算结果以柱状图表示进行对比, 如图9所 示. 在吸附能均为负值的情况下, 绝对值越大表示吸附 越强, 以下吸附能大小比较均指绝对值. 比较不同价态 无机磷在各金属氧化物基底表面的吸附能，正磷的吸 附总体明显强于亚磷和次磷，正磷在 $\mathrm{Fe}_{2} \mathrm{O}_{3}, \mathrm{ZrO}_{2}$,

表 1 不同价态无机磷在金属氧化物基底上的吸附能 $(\mathrm{eV})$

Table 1 Adsorption energies (in $\mathrm{eV}$ ) of inorganic $\mathrm{P}(\mathrm{I} / \mathrm{III} / \mathrm{V}$ ) on metal oxide substrates

\begin{tabular}{ccccc}
\hline 基底 & $\begin{array}{c}\text { 晶面/切割方 } \\
\text { 向 }\end{array}$ & $\mathrm{H}_{3} \mathrm{PO}_{2}$ & $\mathrm{H}_{3} \mathrm{PO}_{3}$ & $\mathrm{H}_{3} \mathrm{PO}_{4}$ \\
\hline$\alpha-\mathrm{Fe}_{2} \mathrm{O}_{3}$ & $(104)$ & -0.39 & -0.15 & -0.85 \\
$\alpha-\mathrm{Fe}_{2} \mathrm{O}_{3}$ & $(110)$ & -0.95 & -0.54 & -1.13 \\
无定形 $\mathrm{Fe}_{2} \mathrm{O}_{3}$ & $(104)$ & -0.45 & 0.57 & -0.30 \\
无定形 $\mathrm{Fe}_{2} \mathrm{O}_{3}$ & $(110)$ & -0.50 & -1.10 & -1.54 \\
$\mathrm{~m}-\mathrm{ZrO}_{2}$ & $(111)$ & -1.02 & -0.53 & -1.04 \\
无定形 $\mathrm{ZrO}_{2}$ & $(111)$ & -1.06 & -1.73 & -6.68 \\
$\mathrm{~h}-\mathrm{La}_{2} \mathrm{O}_{3}$ & $(101)$ & -2.00 & -3.31 & -3.66 \\
无定形 $\mathrm{La}_{2} \mathrm{O}_{3}$ & $(101)$ & -8.39 & -7.87 & -14.33 \\
\hline
\end{tabular}

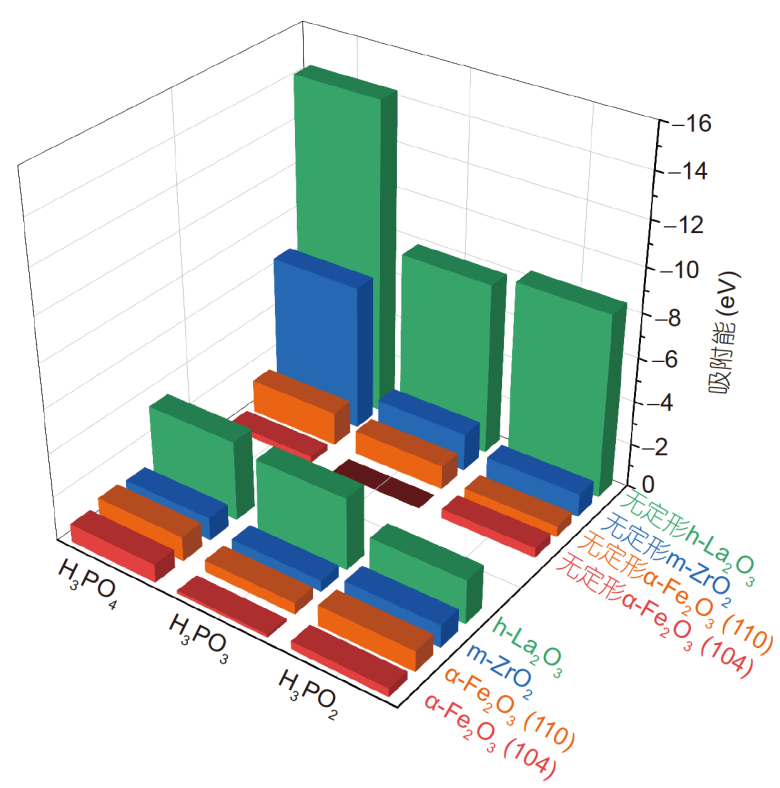

图 9 (网络版彩图)不同价态无机磷在金属氧化物基底上的 吸附能比较

Figure 9 (Color online) Comparison of adsorption energies of inorganic $\mathrm{P}(\mathrm{I} / \mathrm{III} / \mathrm{V})$ on metal oxide substrates.

$\mathrm{La}_{2} \mathrm{O}_{3}$ 三种金属氧化物基底上的吸附能分别是次/亚磷 的 $0.7 \sim 5.6$ 倍、1.0 6.3倍、1.1 1.8倍.

以 $0.5 \mathrm{eV}$ 为物理吸附和化学吸附的界限, 吸附能 (绝对值)大于 $0.5 \mathrm{eV}$ 时一般认为属于化学吸附 ${ }^{[26]}$, 则上 述所有 $\mathrm{Fe}_{2} \mathrm{O}_{3}$ 基底吸附正磷均属于化学吸附, 这与文献 中的结论一致. $\mathrm{Ta}$ 等人 ${ }^{[27]}$ 使用VASP计算了 $\mathrm{Fe}_{2} \mathrm{O}_{3}$ 吸附 正磷酸钠 $\left(\mathrm{Na}_{3} \mathrm{PO}_{4}\right)$ 的情况, 发现该吸附也属于化学吸 附; Lalley等人 ${ }^{[28]}$ 通过实验的方法说明了氧化铁吸附 磷酸盐的吸附属于化学吸附. 理论计算和实验研究均 表明，氧化铁可专属吸附正磷，根据化学吸附的特点， 该吸附过程抗基质干扰能力强. 相较正磷, 次磷、亚磷 在氧化铁基底上的吸附能较小, 多属于物理吸附. 金属 氧化物对水中一些常见离子的吸附能与之相当或更 高, 例如, Paul等人 ${ }^{[29]}$ 通过高斯软件计算得到 $\mathrm{Al}$ 和 $\mathrm{Fe}$ 氧 化物团簇对硫酸根的吸附能在 $-19 \sim-124 \mathrm{~kJ} / \mathrm{mol}$ $(-0.20 \sim-1.29 \mathrm{eV})$ 之间. 由此, 次/亚磷在金属氧化物基 底上的吸附能与硫酸根相比并无明显优势, 可预见在 复杂基质背景下的实际吸附过程中将受到水中共存离 子的竞争干扰.

$\mathrm{ZrO}_{2}$ 和 $\mathrm{La}_{2} \mathrm{O}_{3}$ 吸附次/亚/正磷的吸附能均大于 $0.5 \mathrm{eV}$, 按照上述经验值判断均属于化学吸附, 但吸附 正磷的吸附能更大. Luo等人 ${ }^{[30]}$ 研究了与磷同族的不 
同价态的无机砷在 $\mathrm{m}-\mathrm{ZrO}_{2}$ 上的吸附情况, 使用Materials Studio中的DMol3 模块计算了 $\mathrm{m}-\mathrm{ZrO}_{2}$ 对 $\mathrm{As}(\mathrm{III})$ 和 $\mathrm{As}(\mathrm{V})$ 的吸附能分别为 0.12 和 $1.90 \mathrm{eV}$, 即 $\mathrm{m}-\mathrm{ZrO}_{2}$ 对 $\operatorname{As}(\mathrm{V})$ 的吸附能显著大于As(III), 本文对不同价态无机 磷的探讨结果与此类似.

比较晶型和无定形金属氧化物对次/亚/正磷的吸 附能, 可见无定形金属氧化物的吸附能普遍大于晶型 金属氧化物, 以 $\mathrm{ZrO}_{2}$ 和 $\mathrm{La}_{2} \mathrm{O}_{3}$ 尤为明显. 无定形 $\mathrm{ZrO}_{2}$ 对 次/亚/正磷的吸附能是 $\mathrm{m}-\mathrm{ZrO}_{2}$ 的 $1.0 \sim 6.4$ 倍, 无定形 $\mathrm{La}_{2} \mathrm{O}_{3}$ 是h $-\mathrm{La}_{2} \mathrm{O}_{3}$ 的 2.4 4.2倍. 总体而言, 次/亚/正磷在 无定形金属氧化物基底上的吸附能均值是相应晶型金 属氧化物基底上的 3.1 倍. 无定形金属氧化物吸附能更 大可归因于无定形金属氧化物通常存在缺陷配位及伴 随的氧空位 ${ }^{[31,32]}$. 本研究中, $\alpha-\mathrm{Fe}_{2} \mathrm{O}_{3}, \mathrm{~h}-\mathrm{La}_{2} \mathrm{O}_{3}, \mathrm{~m}-\mathrm{ZrO}_{2}$ 中 Fe, $\mathrm{La}, \mathrm{Zr}$ 的配位数分别为 $6,7,7$, 相应的无定形金属 氧化物中部分 $\mathrm{Fe}, \mathrm{La}, \mathrm{Zr}$ 的配位数分别为 $5,6,6$, 出现缺 陷配位现象, 从而更有利于吸附.

比较三种金属氧化物, 对次/亚/正磷的吸附能顺序 为: $\mathrm{La}_{2} \mathrm{O}_{3}>\mathrm{ZrO}_{2}>\mathrm{Fe}_{2} \mathrm{O}_{3} . \mathrm{La}_{2} \mathrm{O}_{3}$ 是 $\mathrm{Fe}_{2} \mathrm{O}_{3}$ 的2.1 47.5倍, 是 $\mathrm{ZrO}_{2}$ 的2.0 7.4倍, $\mathrm{ZrO}_{2}$ 是 $\mathrm{Fe}_{2} \mathrm{O}_{3}$ 的0.9 22.2倍. Wang等 人 ${ }^{[33]}$ 采用实验比较了 $\mathrm{ZrO}_{2}$ 与另一种铁氧化物 $\mathrm{Fe}_{3} \mathrm{O}_{4}$ 吸 附磷酸盐的吸附等温线, 发现 $\mathrm{ZrO}_{2}$ 的吸附能力更强, 且 制备的4种不同摩尔比的 $\mathrm{Fe}_{3} \mathrm{O}_{4}$ 与 $\mathrm{ZrO}_{2}$ 复合的材料中, 摩尔比为 $1: 2,1: 4$ 的材料吸附明显优于摩尔比为 $9: 1,4: 1$ 的材料, 说明 $\mathrm{ZrO}_{2}$ 对吸附的贡献更大. $\mathrm{La}_{2} \mathrm{O}_{3}$ 的吸附能远 大于其他两种金属氧化物, 这与文献广泛报道的 La化合 物普遍对正磷有较好的吸附性能一致 ${ }^{[18,34 \sim 37]} ; \mathrm{La}_{2} \mathrm{O}_{3}$ 吸附 正磷的吸附能数值大于一般化学吸附, 亦符合文献 [38 40]报道的La化合物吸附正磷表现为化学吸附的实 验研究结论. 诸多实验研究 ${ }^{[41-43]}$ 通过XRD等实验表征发 现镧氧化物吸附正磷后生成磷酸镧晶体, 即发生了化学 反应, 由此可见, 实验和本文理论计算都一致证明氧化
锞与正磷之间的极强的化学作用. $\mathrm{La}_{2} \mathrm{O}_{3}$ 吸附次/亚磷的 吸附能绝对值也较大, 说明具有专属吸附的可能性, 但 目前尚未有相关研究的报道, 有待实验验证.

\section{4 结论}

（1）通过熔化-淬冷方法构建了无定形金属氧化物 模型, 经XRD与RDF验证, 无定形金属氧化物构建成 功. 对晶型金属氧化物表面、无定形金属氧化物表 面、次/亚/正磷、吸附构型都成功得到优化构型, 并 计算了高精度单点能.

(2) 无论晶型或无定形, 三种金属氧化物对正磷的 吸附能普遍大于次/亚磷 (吸附能绝对值为 $0.7 \sim 6.3$ 倍), 表明金属氧化物更容易吸附正磷, 对次/亚磷吸附相对 较弱. 与水中一些常见离子(如硫酸根)相比, 金属氧化 物对次/亚磷的吸附能无明显优势, 加上次/亚磷在水中 的浓度通常较低, 在实际吸附过程中次/亚磷容易受到 竞争离子的影响.

(3) 无定形金属氧化物对次/亚/正磷的吸附能(绝 对值)普遍大于相应的晶型金属氧化物. 无定形 $\mathrm{Fe}_{2} \mathrm{O}_{3}$ 吸附能绝对值均值比晶型大 $0.11 \mathrm{eV}$; 无定形 $\mathrm{ZrO}_{2}$ 和 $\mathrm{La}_{2} \mathrm{O}_{3}$ 的吸附能是晶型的1.0 6.4倍, 可归因于无定形 氧化物缺陷配位现象和氧空位的作用.

(4) 三种金属氧化物相比, 对次/亚/正磷的吸附能 (绝对值)顺序为: $\mathrm{La}_{2} \mathrm{O}_{3}>\mathrm{ZrO}_{2}>\mathrm{Fe}_{2} \mathrm{O}_{3} . \quad \mathrm{La}_{2} \mathrm{O}_{3}$ 基底上 次/亚/正磷的吸附能是 $\mathrm{Fe}_{2} \mathrm{O}_{3}$ 的 2.1 47.5倍, 是 $\mathrm{ZrO}_{2}$ 的 2.0 7.4倍, 体现出La-P之间强烈的化学作用.

(5) 三种金属氧化物吸附正磷普遍属于化学吸附. $\mathrm{Fe}_{2} \mathrm{O}_{3}$ 吸附次/亚磷既有物理吸附, 也有化学吸附; $\mathrm{ZrO}_{2}$ 和 $\mathrm{La}_{2} \mathrm{O}_{3}$ 吸附次/亚磷吸附能数值上表现为化学吸附. 其中, $\mathrm{La}_{2} \mathrm{O}_{3}$ 吸附次/亚磷的吸附能绝对值高于 $2.00 \mathrm{eV}$, 表明具有在复杂基质背景下实现专属吸附的可能性.

致谢 本文的数值计算是在南京大学高性能计算中心的计算集群上完成的.

\section{参考文献}

1 Chen X F, Chuai X M, Yang L Y. Status quo, historical evolution and causes of eutrophication in lakes in typical lake regions of China (in Chinese). J Ecol Rural Environ, 2014, 30: 438-443 [陈小锋, 揣小明, 杨柳燕. 中国典型湖区湖泊富营养化现状、历史演变趋势及成因分析. 生态与农村环境学报, 2014, 30: 438-443] 
2 Li Y Y. Research on eutrophication threshold and limiting factors in Miyun reservoir (in Chinese). Dissertation for Master's Degree. Zhengzhou: Henan University, 2017 [李亚永. 密云水库富营养化阈值与限制因子研究. 硕士学位论文. 郑州: 河南大学, 2017]

$3 \mathrm{Xu} \mathrm{W}$ Y. Study on speciation and removal of phosphorus in tail water from municipal wastewater treatment plant (in Chinese). Dissertation for Master's Degree. Hangzhou: Zhejiang University of Technology, 2009 [徐伟勇. 城市污水处理厂尾水中磷的形态分析及除磷研究. 硕士学位 论文. 杭州: 浙江工业大学, 2009]

4 Liang S, Zheng W, Zhu L, et al. One-step treatment of phosphite-laden wastewater: A single electrochemical reactor integrating superoxide radical-induced oxidation and electrocoagulation. Environ Sci Technol, 2019, 53: 5328-5336

5 Qiu H, Geng J, Ren H, et al. Phosphite flux at the sediment-water interface in northern Lake Taihu. Sci Total Environ, 2016, 543: 67-74

6 Jiang X X, Shen W. The Fundamentals and Practice of Electroless Plating (in Chinese). Beijing: National Defense Industry Press, 2000 [姜晓霞, 沈伟. 化学镀理论及实践. 北京: 国防工业出版社, 2000]

7 Zhou J, Liu P, Liu Z, et al. Simultaneous recovery of phosphorus with nickel purification in nickel-plating wastewater via Fe/C activated $\mathrm{H}_{2} \mathrm{O}_{2}$ oxidation. Chem Eng J, 2020, 381: 122702

8 Hesselmann R P X, Werlen C, Hahn D, et al. Enrichment, phylogenetic analysis and detection of a bacterium that performs enhanced biological phosphate removal in activated sludge. Systatic Appl Microbiol, 1999, 22: 454-465

9 Kong Y H, Beer M, Rees G N, et al. Functional analysis of microbial communities in aerobic-anaerobic sequencing batch reactors fed with different phosphorus/carbon (P/C) ratios. Microbiology, 2002, 148: 2299-2307

10 Song D, Yang S B. Study on influence factors of phosphorous removal by chemical method in sewage treatment system (in Chinese). J Anhui Agric Sci, 2013, 41: 7645-7648 [宋丹, 杨肃博. 污水处理系统中化学除磷效果影响因素分析. 安徽农业科学, 2013, 41: 7645-7648]

11 Ayoub G M, Koopman B, Pandya N. Iron and aluminum hydroxy (oxide) coated filter media for low-concentration phosphorus removal. Water Environ Res, 2001, 73: 478-485

12 Genz A, Kornmüller A, Jekel M. Advanced phosphorus removal from membrane filtrates by adsorption on activated aluminium oxide and granulated ferric hydroxide. Water Res, 2004, 38: 3523-3530

13 Pasek M, Herschy B, Kee T P. Phosphorus: A case for mineral-organic reactions in prebiotic chemistry. Orig Life Evol Biosph, 2015, 45: 207218

14 Qiu H, Geng J, Shen C, et al. Aquatic photooxidation of phosphite in the presence of ferric and oxalate ions. Chem Eng J, 2015, 269: 408-415

15 Guan W, Tian S, Ma N, et al. An electrochemical method through hydroxyl radicals oxidation and deposition of ferric phosphate for hypophosphite recovery. J Colloid Interface Sci, 2018, 516: 529-536

16 Zhang H, Elskens M, Chen G, et al. Phosphate adsorption on hydrous ferric oxide (HFO) at different salinities and pHs. Chemosphere, 2019, 225: 352-359

17 Liu H, Sun X, Yin C, et al. Removal of phosphate by mesoporous $\mathrm{ZrO}_{2}$. J Hazard Mater, 2008, 151: 616-622

18 Ding W M, Huang X, Zhang L P. Removal of phosphorus from aqueous solution by lanthanum hydrate (in Chinese). Environ Sci, 2003, 24: 110113 [丁文明, 黄霞, 张力平. 水合氧化镧吸附除磷的试验研究. 环境科学, 2003, 24: 110-113]

19 Gao W, Chen Y, Li B, et al. Determining the adsorption energies of small molecules with the intrinsic properties of adsorbates and substrates. Nat Commun, 2020, 11: 1196

20 Shan C, Liu H, Hua M, et al. Enhanced Fenton-like oxidation of As(III) over Ce-Ti binary oxide: A new strategy to tune catalytic activity via balancing bimolecular adsorption energies. Environ Sci Technol, 2020, 54: 5893-5901

21 Pandey A, Scherich H, Drabold D A. Density functional theory model of amorphous zinc oxide (a-ZnO) and a- $\mathrm{X}_{0.375} \mathrm{Z}_{0.625} \mathrm{O}(\mathrm{X}=\mathrm{Al}, \mathrm{Ga}$ and In). J Non-Crystalline Solids, 2017, 455: 98-101

22 Momma K, Izumi F. VESTA 3 for three-dimensional visualization of crystal, volumetric and morphology data. J Appl Crystlogr, 2011, 44: 12721276

23 Stumm W, Kummert R, Sigg L. A ligand-exchange model for the adsorption of inorganic and organic-ligands at hydrous oxide interfaces. Croat Chem Acta, 1980, 53: 291-312

24 Kresse G, Furthmüller J. Efficient iterative schemes for ab initio total-energy calculations using a plane-wave basis set. Phys Rev B, 1996, 54: $11169-11186$

25 Perdew J P, Burke K, Ernzerhof M. Generalized gradient approximation made simple. Phys Rev Lett, 1996, 77: 3865-3868

26 Spanjaard D, Desjonquères M C. Electronic Theory of Chemisorption. In: Bortolani V, March N H, Tosi M P, eds. Interaction of Atoms and 
Molecules with Solid Surfaces. Physics of Solids and Liquids. Boston, MA: Springer, 1990

27 Ta H T T, Tieu A K, Zhu H, et al. Chemical origin of sodium phosphate interactions on iron and iron oxide surfaces by first principle calculations. J Phys Chem C, 2017, 122: 635-647

28 Lalley J, Han C, Li X, et al. Phosphate adsorption using modified iron oxide-based sorbents in lake water: Kinetics, equilibrium, and column tests. Chem Eng J, 2016, 284: 1386-1396

29 Paul K W, Kubicki J D, Sparks D L. Quantum chemical calculations of sulfate adsorption at the Al- and Fe-(Hydr)oxide- $\mathrm{H}_{2} \mathrm{O}$ interface estimation of Gibbs free energies. Environ Sci Technol, 2006, 40: 7717-7724

30 Luo J, Luo X, Hu C, et al. Zirconia $\left(\mathrm{ZrO}_{2}\right)$ embedded in carbon nanowires via electrospinning for efficient arsenic removal from water combined with DFT studies. ACS Appl Mater Interfaces, 2016, 8: 18912-18921

31 Albuquerque A R, Bruix A, dos Santos I M G, et al. DFT study on Ce-doped anatase $\mathrm{TiO}_{2}$ : Nature of $\mathrm{Ce}^{3+}$ and $\mathrm{Ti}^{3+}$ centers triggered by oxygen vacancy formation. J Phys Chem C, 2014, 118: 9677-9689

32 Mavračić J, Mocanu F C, Deringer V L, et al. Similarity between amorphous and crystalline phases: The case of TiO 2 . J Phys Chem Lett, 2018, 9: 2985-2990

33 Wang Z, Xing M, Fang W, et al. One-step synthesis of magnetite core/zirconia shell nanocomposite for high efficiency removal of phosphate from water. Appl Surf Sci, 2016, 366: 67-77

34 Shin E W, Karthikeyan K G, Tshabalala M A. Orthophosphate sorption onto lanthanum-treated lignocellulosic sorbents. Environ Sci Technol, 2005, 39: 6273-6279

35 Wu R S S, Lam K H, Lee J M N, et al. Removal of phosphate from water by a highly selective La(III)-chelex resin. Chemosphere, 2007, 69: 289294

36 Yang J, Yuan P, Chen H Y, et al. Rationally designed functional macroporous materials as new adsorbents for efficient phosphorus removal. J Mater Chem, 2012, 22: 9983-9990

37 Li B, Ning P, Chen Y B, et al. Nitrogen and phosphate removal by activated zeolite with lanthana (in Chinese). J Wuhan Univ Tech, 2005, 27: 5659 [李涁, 宁平, 陈玉保, 等. 氧化镧改性沸石除磷脱氮研究. 武汉理工大学学报, 2005, 27: 56-59]

38 Liu D, Zhou W, Wu J. Kinetic behavior of elemental mercury sorption on cerium- and lanthanum-based composite oxides. Surf Rev Lett, 2019, 26: 1850141

39 Yuan L, Qiu Z, Lu Y, et al. Development of lanthanum hydroxide loaded on molecular sieve adsorbent and mechanistic study for phosphate removal. J Alloys Compd, 2018, 768: 953-961

40 Xiang C, Ji Q, Zhang G, et al. In situ creation of oxygen vacancies in porous bimetallic La/Zr sorbent for aqueous phosphate: Hierarchical pores control mass transport and vacancy sites determine interaction. Environ Sci Technol, 2019, 54: 437-445

41 Zhang Y, Pan B, Shan C, et al. Enhanced phosphate removal by nanosized hydrated La(III) oxide confined in cross-linked polystyrene networks. Environ Sci Technol, 2016, 50: 1447-1454

42 Jing X, Wang Y, Chen L, et al. Free-standing large-mesoporous silica films decorated with lanthanum as new adsorbents for efficient removal of phosphate. J Mol Liquids, 2019, 296: 111815

43 Qiu H, Liang C, Yu J, et al. Preferable phosphate sequestration by nano-La(III) (hydr)oxides modified wheat straw with excellent properties in regeneration. Chem Eng J, 2017, 315: 345-354 


\title{
Adsorption of inorganic phosphorus of different valences on metal oxides: A first-principles study
}

\author{
HENG ZhongXuan ${ }^{1}$, SHAN Chao ${ }^{1,2}$, HUA Ming ${ }^{1,2}$ \& PAN BingCai ${ }^{1,2}$ \\ ${ }^{1}$ State Key Laboratory of Pollution Control and Resource Reuse, School of Environment, Nanjing University, Nanjing 210023, China; \\ ${ }^{2}$ Research Center for Environmental Nanotechnology (ReCENT), Nanjing University, Nanjing 210023, China
}

Phosphorus is the key factor limiting eutrophication. Although the prevailing decontamination technologies for the removal of orthophosphate are relatively mature, the advanced treatment of hypophosphite and phosphite has not attracted sufficient attention. Metal oxides are commonly employed as adsorbents for phosphate removal and are of great significance to the investigation of the adsorption behaviors and mechanisms of inorganic phosphorus of different valences on metal oxides. In this study, bulk phase and slab models of crystalline and amorphous $\mathrm{Fe}_{2} \mathrm{O}_{3}, \mathrm{ZrO}_{2}, \mathrm{La}_{2} \mathrm{O}_{3}$ were constructed, and the adsorption configurations and energies of hypophosphite, phosphite, and orthophosphate on the above substrates were determined using first-principles calculations. Results show that hypophosphite, phosphite, and orthophosphate are adsorbed on the metal oxides mainly via the formation of M-O-P bonds $(\mathrm{M}=$ a metal atom) and hydrogen bonds $(\mathrm{O}-\mathrm{H})$. Comparison of adsorption energies revealed that orthophosphate is mainly chemisorbed on the surface of metal oxides and its adsorption energies are generally 0.7-6.3 times greater than those of hypophosphite/phosphite. This finding reflects the specific adsorption of orthophosphate on metal oxides. By contrast, the adsorption energies of hypophosphite and phosphite are relatively low, which implies weak adsorption ability due to the competitive interference induced by coexisting ions. The adsorption energies on amorphous metal oxides are, on average, 3.1 times greater than those on their crystalline analogues. The adsorption energies on the investigated metal oxides followed the order $\mathrm{Fe}_{2} \mathrm{O}_{3}<\mathrm{ZrO}_{2}<\mathrm{La}_{2} \mathrm{O}_{3}$. The adsorption energies on La oxides were 2.0-47.5 times greater than those of iron/zirconium oxides, indicating strong bonding between $\mathrm{La}$ and $\mathrm{PO}_{x}$ groups. This study is helpful for the selection and development of water decontamination technologies for the in-depth removal of phosphorus.

adsorption, phsphorus speciation, metal oxides, first-principles calculation, adsorption energy

doi: 10.1360/SST-2020-0281 